\title{
Does pain hypervigilance further impact the lack of habituation to pain in individuals with chronic pain? A cross-sectional pain ERP study
}

This article was published in the following Dove Press journal: Journal of Pain Research

\author{
Catherine J Vossen' \\ Rosan Luijcks ${ }^{2}$ \\ Jim van Os ${ }^{2,3}$ \\ Elbert A Joosten' \\ Richel Lousberg ${ }^{2}$ \\ 'Department of Anaesthesiology \\ and Pain Medicine, ${ }^{2}$ Department of \\ Psychiatry \& Psychology, Maastricht \\ University Medical Centre, Maastricht, \\ The Netherlands; ${ }^{3}$ King's College \\ London, King's Health Partners, \\ Department of Psychosis Studies, \\ Institute of Psychiatry, London, UK
}

Correspondence: Catherine J Vossen Department of Anaesthesiology and Pain Medicine, Maastricht University Medical Centre, P.O. Box 5800, 6202 AZ Maastricht, The Netherlands

$\mathrm{Tel}+3$ I 433877455

Fax +31 433875457

Email c.vossen@mumc.nl
Aim: In chronic pain, habituation is believed to be impaired, and pain hypervigilance can enhance the pain experience. The goal of this study was to determine whether pain hypervigilance further worsens habituation of event-related potentials, measured in a pain-rating protocol of 25 painful somatosensory electrical stimuli, in patients with chronic pain.

Methods: Pain hypervigilance was assessed with the Pain Vigilance Awareness Questionnaire and analyzed using the event-related fixed interval areas multilevel technique, which enables one to study within-session habituation. In a cohort of 111 participants, 33 reported chronic pain. This chronic pain group was compared with 33 pain-free individuals, matched for age and sex. Results: The relationship between pain status and habituation was not moderated by pain hypervigilance. Chronic pain status affected linear habituation and dishabituation (quadratic function) from 220 to $260 \mathrm{~ms}$ for nearly all electrodes, and from 580 to $640 \mathrm{~ms}$ for frontal electrodes. The effect of pain hypervigilance on habituation was observed primarily from 480 to $820 \mathrm{~ms}$ poststimulus for right-sided and central electrodes.

Conclusion: Pain hypervigilance and chronic pain independently influence habituation to painful stimuli - although not synergistically. To confirm that these effects are mediated by separate pathways, further research is required, in which electroencephalography is combined with other modalities with adequate spatial resolution, such as functional magnetic resonance imaging.

Keywords: event-related fixed-interval area, ERFIA, multilevel analysis, habituation, pain hypervigilance, chronic pain

\section{Introduction}

The perception of pain does not result solely from incoming noxious information; other factors, such as cognition, emotions, and attention, affect the perception of pain and its subsequent behavior. For instance, acute pain can serve as a protective mechanism, signaling the body to rest, allowing healing to commence. However, in other circumstances, such as in dangerous situations, pain must be ignored to meet a higher order goal that is prioritized over momentary pain. ${ }^{1}$ Thus, the brain modulates this perception through the facilitation or inhibition of nociceptive input. As a result, pain perception arises from a balance between nociceptive and antinociceptive mechanisms.

In chronic pain - pain that persists or recurs for more than 3-6 months - acute warnings are less functional. ${ }^{2}$ Phenomena such as peripheral and central sensitization facilitate the development of chronic pain. ${ }^{3}$ On the other hand, inhibitory pathways, among which is habituation, may protect against chronic pain. ${ }^{4}$ There are indications that the balance between nociceptive and antinociceptive processes is distorted. ${ }^{5,6}$ One of the inhibitory (antinociceptive) pathways that is believed to be impaired in 
chronic pain is habituation. Habituation is a decrease in the behavioral response that occurs after repetition of a variety of types of stimuli and is considered to be an elementary form of learning. ${ }^{7-9}$ Habituation to painful stimuli might protect against the development of chronic pain..$^{5,10,11}$ Habituation is thought to serve as an attentional filter in pain. ${ }^{12}$ Impaired habituation has been demonstrated in several chronic pain populations, such as in groups with low-back pain, migraine, and fibromyalgia. ${ }^{13-16}$

Furthermore, attention is believed to facilitate nociceptive mechanisms. Paying attention to nociceptive input renders it more painful. Thus, a cognitive factor that has been proposed to increase pain perception is a preoccupation or heightened attention to pain, also known as pain hypervigilance. ${ }^{17}$ Attention to pain can be measured by the Pain Hypervigilance and Awareness Questionnaire (PVAQ). ${ }^{18}$ This questionnaire is validated for healthy subjects as well as several chronic pain populations. It is assumed that hypervigilance to painrelated stimuli increases pain experiences. ${ }^{18-21}$ Moreover, pain hypervigilance has been suggested to predispose healthy individuals to chronic pain. ${ }^{22}$

Based on their superior temporal resolution, eventrelated potentials (ERPs) are suitable for the assessment of habituation to pain. ERPs are time-locked cortical responses to (painful) stimuli that are derived from ongoing electroencephalography (EEG) activity. ERPs are EEG segments (so-called epochs) around time markers of delivered stimuli in the ongoing EEG. Recently, the event-related fixed-interval area (ERFIA) multilevel technique was introduced to analyze ERPs, which have several advantages in the study of habituation. ${ }^{23}$ Multilevel analyses of ERPs allow one to examine the within-session time course - that is, habituation-over trials, whereas other techniques merely analyze betweensession habituation. ${ }^{24}$

In addition, person-by-time effects and their nonlinear properties can be modeled. For example, in studying various forms of habituation, a linear function for stimulus number can be incorporated into the model to analyze linear declines over 25 stimuli. By modeling a parabolic (quadratic) function, an initial decrease and subsequent rise in the response over 25 stimuli can be examined, representing impaired habituation. In this article, we use the terms "linear habituation" for the linear function and "dishabituation" for the quadratic function.

By subdividing the poststimulus epoch into small fixedinterval areas (ERFIAs), the time course over trials can be studied for various poststimulus latencies. ${ }^{23}$ For instance, the manifestation of habituation might differ at $300 \mathrm{~ms}$ versus
$400 \mathrm{~ms}$ poststimulus. Two ERP studies analyzed several aspects of habituation, chronic pain, and pain hypervigilance using the ERFIA multilevel method. The first study, conducted in individuals with chronic low-back pain and painfree controls, reported that linear and nonlinear habituation depended on chronic pain status over a broad poststimulus range. ${ }^{13}$ The second trial demonstrated that pain hypervigilance impacts the cortical processing of painful stimuli and its habituation in pain-free subjects (Vossen, submitted 2016).

Based on this collective evidence, chronic pain and habituation are linked, and pain hypervigilance and habituation are associated in pain-free subjects. However, the extent to which chronic pain, pain hypervigilance, and habituation are related is unknown. Thus, we hypothesize that the impaired habituation that is observed in chronic pain is affected by pain hypervigilance, wherein the attention to pain increases.

In this study, we tested the hypothesis that the effects of pain status on habituation are moderated by pain hypervigilance, as measured with the PVAQ, expecting that greater pain hypervigilance in chronic pain further impairs habituation of event-related EEGs as compared with pain-free hypervigilant subjects. In addition, the relationship between pain hypervigilance, chronic pain, and habituation might become apparent at the cortical and behavioral levels. Thus, we speculated that higher pain hypervigilance scores and chronic pain status increase the pain-intensity report of stimuli, as measured on a numerical rating scale (NRS), and alters the course of NRS scores over 25 consecutive trials.

\section{Methods \\ Participants}

This study was a subset of a larger project that evaluated psychophysiological reactivity as a predictor of change in pain complaints. The original study was approved by the medical ethics committee of Maastricht University Medical Centre, Maastricht, the Netherlands (NL40284.068.12/METC 12-3-015). Study participants consisted of a sample of the general population of Maastricht who were recruited using flyers that were distributed throughout five neighborhoods in Maastricht. The inclusion criteria were age between 18 and 65 years and a good understanding of Dutch. The exclusion criteria were as follows: 1) structural use of psychoactive medications, such as antipsychotics, antidepressants, antiepileptics, and anxiolytics, during the past year; 2) regular use of alcohol >10 U/day during the past year; 3) epilepsy; 4) psychotic disorder; 5) visual or hearing disability; and 6) analphabetism or dyslexia. Subjects were only included if they did not consume any alcoholic beverages the evening 
prior to the experiment and refrained from caffeine-containing beverages 3 hours before the start of the experiment.

Before the experiment, written informed consent was obtained. Subjects were rewarded with $€ 50$ for their participation after completion of the entire experiment.

Out of the 111 subjects in the original study, 33 had chronic pain complaints, based on the Short-Form Health Survey (SF-36) and Brief Pain Inventory (BPI) questionnaires. These 33 patients were frequency-matched for age and sex with pain-free controls from the same dataset. The pain-free participants were selected according to the following criteria: 1) no pain complaints at the time of the experiment, 2) no pain complaints in the 6 months before the experiment, and 3) no chronic use of pain medication in the previous 6 months.

\section{Questionnaires}

Prior to the habituation experiment, subjects were asked to complete three questionnaires in an adjacent room in the laboratory: 1) SF-36, consisting of 36 items, to assess their general health status and, in particular, the bodily pain subscale, ${ }^{25}$ 2) Brief Pain Inventory Short Form (BPI-SF), which comprises nine items on pain complaints, ${ }^{26,27}$ and 3) Pain Vigilance Awareness Questionnaire (PVAQ), which contains 16 items on pain behavior with regard to attention to pain and attention to changes in pain. ${ }^{18}$ The PVAQ consists of 16 items that are rated on a 6-point scale (0-5). A combined sum score after inversion of two questions between 0 and 5 is calculated, with a maximum score of 80 . The Dutch version of the PVAQ has a good internal consistency and a fair test-retest reliability. ${ }^{28,29}$ For this questionnaire, there are no defined cutoff points. Higher scores indicate a higher degree of pain hypervigilance. Additional questions were asked concerning subject characteristics (e.g., age, sex), pain complaints in the past 6 months, location of pain complaints, and medication use.

\section{Electroshocker and stimuli}

An electroshocker (Shocko-100-AA-20, developed by Maastricht Instruments BV and approved for use in experimental studies) was used to apply the electrical stimuli. Electrical pulse stimuli (duration: $10 \mathrm{~ms}$ ) were delivered intracutaneously to the left middle finger, according to a method described by Bromm and Meier. ${ }^{30}$ With a dental gimlet of 1 $\mathrm{mm}$, a small lumen in the epidermis was carefully prepared, ensuring that the procedure was not painful. A concentric gold electrode with a diameter of $0.9 \mathrm{~mm}$ and a $1 \mathrm{~mm}$ extension was attached to the prepared lumen and fixed with tape.
Proximal to the prepared finger, a grounding wrist strap (3M wrist strap, WBB-AFWS61M) was placed around the wrist. Next, sensation and pain thresholds were measured to determine the intensity of the stimuli for the habituation protocol by gradually increasing the intensity of the stimulus, starting at 0 and increasing by steps of $0.05 \mathrm{~mA}$. The first intensity that was consciously experienced was defined as the sensation threshold; the first intensity that was considered painful was taken as the pain threshold. Subjects were asked to say "stop" as soon as they perceived the intensity of the stimulus as painful. This procedure was carried out three times in total to generate a reliable measurement. The maximum stimulus intensity never exceeded $5 \mathrm{~mA}$.

\section{Habituation protocol}

Based on the subject's difference between the sensation and pain thresholds, a stimulus that was $25 \%$ above the pain threshold was calculated as follows:

Delivered habituation stimulus $=$ pain threshold + $0.25^{*}$ (pain threshold - sensation threshold).

The intensity of the habituation stimulus was experienced as painful but, nevertheless, tolerable. The habituation protocol comprised 25 identical stimuli of $10 \mathrm{~ms}$ duration. The interstimulus intervals (ISIs) varied between 9 and 11 seconds. Subjects were informed that they would experience a series of stimuli and were instructed to determine the differences between stimuli. The intensity and number of stimuli were unknown to the subject. Subjects were asked to verbally rate the intensity of each stimulus on a scale from 0 (no sensation) to 100 (the most severe pain imaginable). For standardization purposes, subjects were asked to score the first stimulus as 60 .

\section{EEG measurement}

The EEG measurements took place in an electrically and sound-shielded cubicle $\left(7.1 \mathrm{~m}^{2}\right)$. EEGs were recorded with the BrainAmp Amplifier using BrainVision and sampled at $1000 \mathrm{~Hz}$. Using the international $10-20$ system, $\mathrm{Ag} / \mathrm{AgCl}$ electrodes were placed on Fz, F3, F4, Cz, C3, C4, T3, T4, Pz, P3, P4, Oz, O1, and O2, respectively. ${ }^{31}$ Reference electrodes were placed on the earlobes, and a ground electrode was fixed at Fpz. To measure vertical eye movements, electrooculogram (EOG) electrodes were placed $1 \mathrm{~cm}$ below the midline of the right and left eyes. All electrodes were fixed with conductive paste. 


\section{Data processing}

In Analyzer 2.0 (Brain Products, München, Germany), trials were segmented from the continuous EEG - from 200 $\mathrm{ms}$ before the stimulus to $1000 \mathrm{~ms}$ poststimulus - and were offline band-pass filtered $(0-50 \mathrm{~Hz})$ and baseline-corrected (interval -200 to $0 \mathrm{~ms}$; Figure 1). For each subject and the 25 stimuli within a subject, the data (microvolts) for each millisecond between -200 and $1000 \mathrm{~ms}$ for all electrodes and EOG channels were imported into SPSS 21.0. Subsequently, a multilevel dataset was constructed for each subject, using a syntax file in which the following calculations were made: 1) 20-ms ERFIAs were calculated from 0 to $1000 \mathrm{~ms}$ poststimulus, resulting in 50 ERFIAs per trial per EEG electrode per subject; 2) maximum and minimum values of the EOG channel were selected per 20-ms ERFIA; and 3) questionnaire data were added to the dataset. Next, all cases were added to obtain a full multilevel dataset. Left and right EOG activities were included per 20-ms ERFIA in the analysis as covariates.

\section{Statistical analysis}

Multilevel regression was done separately for each EEG electrode and every 20-ms ERFIA period. The dependent variable in the multilevel model comprised all 20-ms ERFIAs at a particular poststimulus latency from all trials and subjects. The dependent variables, thus consisting of 50 consecutive 20-ms ERFIAs, were assessed for normality. Subjects represented the highest level in the model, and trial number (1-25 stimuli) was the repeated measure within each subject. Based on the assumption that habituation can differ between subjects, random effects, such as a random intercept and a random slope for trial number, were included. An AR-1 covariance structure was used, assuming that trials that were nearer to each other correlated better than those that were further apart.

As in previous studies, linear habituation (trial number) and dishabituation (parabolic relationship, computed as trial*trial) were modeled. ${ }^{13,23} \mathrm{~A}$ linear function with a negative coefficient reflects a linear decline in ERFIAs over 25 stimuli. A quadratic function, in which the parabola opens upward, represents initial habituation, after which sensitization (or dishabituation) occurs over 25 stimuli. To address our research question, the main effects of PVAQ and pain status (chronic pain vs pain-free) on the poststimulus EEG were measured, using the following basic model: ERFIAs of a specific $20-\mathrm{ms}$ range and electrode (ERFIAs ${ }_{20 \text {-ms range, location }}$ ) constituted the dependent variable, which was modeled as a function of the following fixed factors: $\mathrm{PVAQ}_{\text {median split }}$, pain status, trial number, trial ${ }_{\text {quadratic }}$, age, sex, pain threshold, sensation threshold, EOG left, and EOG right.

To answer our research question with regard to whether the impact of pain status on linear and quadratic habituation is influenced by PVAQ score, two 3-way interactions and five 2-way interactions were added to the model: $\mathrm{PVAQ}_{\text {median split }}{ }^{*}$ pain status *habituation linear $\mathrm{PVAQ}_{\text {median split }}$ *pain status *habituation ${ }_{\text {quadratic }}, \mathrm{PVAQ}_{\text {median split }}$ *habituation ${ }_{\text {linear, }}$ $\mathrm{PVAQ}_{\text {median split }} *$ habituation ${ }_{\text {quadratic }}$, pain status*habituation linear , pain status*habituation ${ }_{\text {quadratic }}$, and $\mathrm{PVAQ}_{\text {median split }}{ }^{*}$ pain status.

For the PVAQ variable, the group-specific - instead of overall - median split was used to ensure that the relationship between PVAQ and group was separated.

Analyses were conducted separately for each consecutive 20-ms ERFIA period (0-1000 ms poststimulus) for all 14 cranial locations. The large number of statistical tests necessitated a correction for multiple testing, but the analyses were

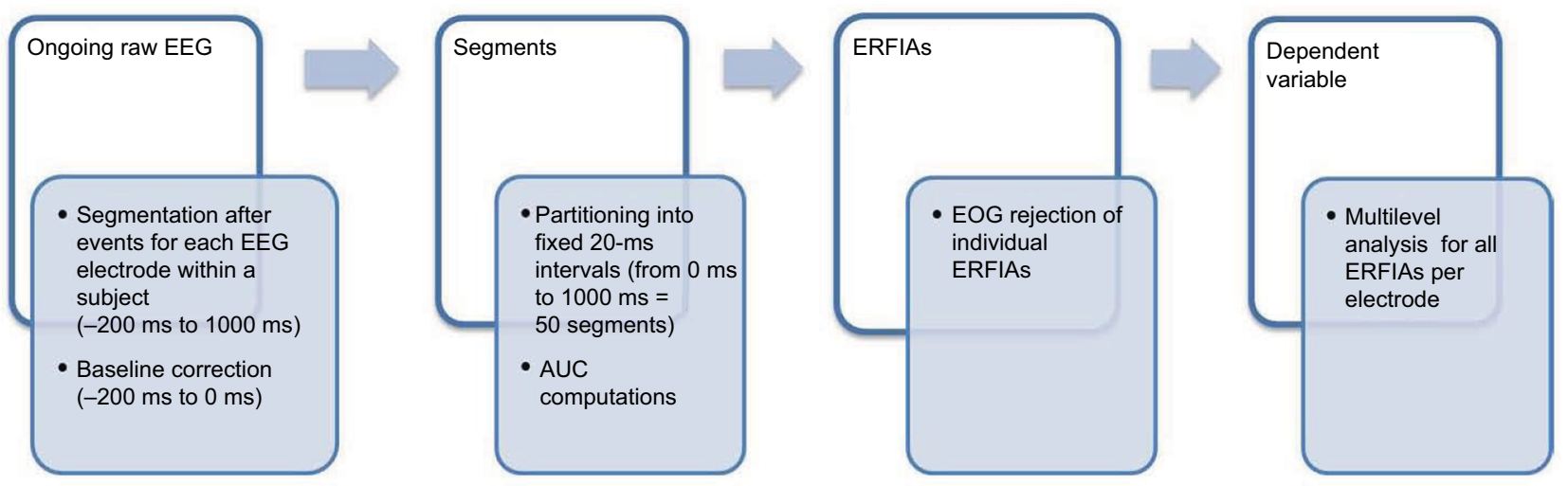

Figure I The processing steps from the "raw" EEG to ERFIAs serving as the dependent variable for multilevel analysis. First, the EEG is partitioned into epochs, and then a baseline correction is made. Next, the segments are divided into 20-ms intervals, and the area under the curve for every interval for all trials is calculated. An EOG rejection is carried out for all ERFIAs separately. In the last step, the valid ERFIAs per fixed interval serve as a dependent variable in the multilevel analysis.

Abbreviations: EEG, electroencephalography; ERFIAs, event-related fixed interval areas; EOG, electrooculogram; AUC, area under the curve. 
exploratory. Thus, we chose not to define a specific $P$-value for statistical significance. Alternatively, we considered only robust effects ( $\geq 3$ consecutive 20 -ms ERFIAs) with $P$-values $\leq 0.05$ to be statistically significant. For low-power interaction effects, we considered robust effects with $P$-values $\leq 0.10$ to be significant.

Results were summarized in ERFIA predictor blots, in which the columns represented 50 consecutive 20 -ms ERFIAs and the rows represented the EEG electrodes of a given predictor. In each row, cells were given a color when $T$-values were $<-2$ or $>2$. Red indicated a positive significant $T$-value, whereas blue denoted a significant negative $T$-value.

The influence of pain status and pain hypervigilance on pain report, as measured on an NRS, was examined, as was their effect on the time course. A basic model was used, in which NRS was the dependent variable and modeled as a function of the following variables: pain status, $\mathrm{PVAQ}_{\text {median split }}$ trial number, trial ${ }_{\text {quadratic }}$, age, and sex. Next, the following interactions were added to analyze the influence of PVAQ and pain status on the time course of the NRS variable: $\mathrm{PVAQ}_{\text {median split }}$ * pain status *habituation linear, $\mathrm{PVAQ}_{\text {median split }}{ }^{*}$ pain status $*$ habituation ${ }_{\text {quadratic }}$,

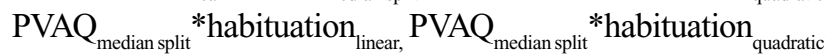
pain status*habituation $_{\text {linear }}$, pain status*habituation quadratic , and $\mathrm{PVAQ}_{\text {median split }}{ }^{*}$ pain status.

For illustrative purposes, an overall course of NRSs across the 25 stimuli was constructed for the two study (chronic pain vs pain-free) groups. All statistical analyses were undertaken with SPSS 21.0 (Figure 2).

\section{Results Subject characteristics}

A total of 111 participants were enrolled from April 2012 to August 2014. Of this cohort, 33 participants reported chronic pain complaints and were matched for age with 33 pain-free individuals. Table 1 summarizes the characteristics of the analyzable participants. The mean PVAQ score was 32

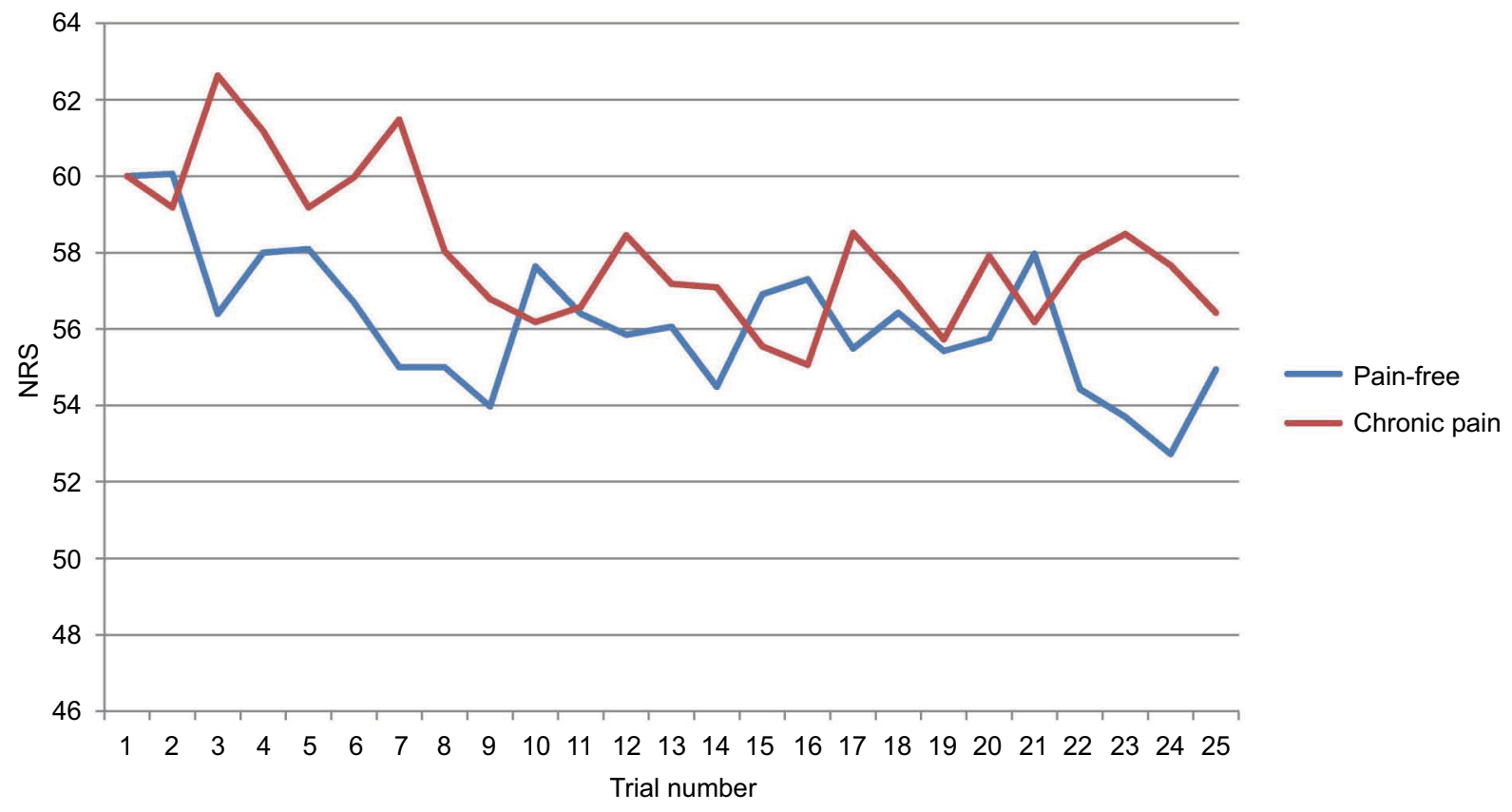

Figure 2 Numerical rating scale (NRS) course of 25 trials for pain-free subjects and participants with chronic pain.

Table I Characteristics of study participants

\begin{tabular}{lllll}
\hline Characteristic & Total & Pain-free & Chronic pain & P-values \\
\hline N & 66 & 33 & 33 & \\
PVAQ score mean (SD) & $31.6(12.1)$ & $28.1(13.1)$ & $35.1(10.1)$ & NS \\
Age years mean (SD) & $42.1(17.0)$ & $39.1(16.7)$ & $45.0(17.0)$ & $\mathrm{NS}$ \\
Sex male/female & $25 / 41$ & $15 / 18$ & $10 / 23$ & $\mathrm{NS}$ \\
Sensation threshold (mA) & $0.29(0.16)$ & $0.26(0.14)$ & $0.32(0.18)$ & $\mathrm{NS}$ \\
Pain threshold (mA) & $0.93(0.52)$ & $0.82(0.52)$ & $1.03(0.52)$ & $\mathrm{NS}$ \\
NRS (of the 25 stimuli) 0-100 (SD) & $57(9.6)$ & $56(10.2)$ & $58(9.1)$ & \\
\hline
\end{tabular}

Abbreviations: PVAQ, Pain Vigilance Awareness Questionnaire; NRS, numerical rating scale; NS, not significant. 
(range: 7-59). The median split for the pain hypervigilance score was 30 for the pain-free group and 35 for the chronic pain group. The mean PVAQ score differed significantly between groups. Table 2 shows the location of pain in the chronic pain participants, based on the BPI; 13 of these participants had pain in more than one area.

\section{Pain hypervigilance and the NRS}

As hypothesized a priori, in the main model, "trial number" was significantly and negatively $(P=0.041)$ associated with NRS, indicating a significant linear decrease of the NRS scores over the course of 25 trials - that is, representing habituation. No significant differences were noted between the chronic pain and pain-free groups, with respect to the course of NRS scores over 25 stimuli. Nor did pain hypervigilance and pain status influence the time course of the NRS scores in the interaction model (Figure 2).

\section{Main influence of chronic pain status and pain hypervigilance on the ERP}

In the primary multilevel model, chronic pain status had a robust, significant positive main effect $(P \leq 0.05)$ at three or more consecutive ERFIAs between 300 and $400 \mathrm{~ms}$ for all electrodes, except for P4 and the occipital locations (Figure 3A). This means that ERFIAs in this region are greater for the chronic pain group compared to the pain-free subjects. With regard to pain hypervigilance, a significant positive association was observed only for F3 from 940 to $980 \mathrm{~ms}$ (Figure 3B).

\section{Main influence of habituation on the ERP}

In the main model, significant effects of both linear habituation and quadratic habituation were seen in a broad range of the ERP for all electrodes. Linear habituation was seen from approximately 160 to $460 \mathrm{~ms}$ and quadratic habituation from 180 to $480 \mathrm{~ms}$ (Figure 3C and D). In addition, a late significant effect of linear and quadratic habituations was observed from 580 to $660 \mathrm{~ms}$ for the Fz and F3 (Figure 3C).

\section{Interaction effects of chronic pain, pain hypervigilance, and habituation on the ERP}

The association between chronic pain status and linear and quadratic habituation did not depend on pain hypervigilance - that is, the two 3-way interactions (chronic pain status *linear habituation*PVAQ and chronic pain status *quadratic habituation*PVAQ) were not significant. In general, the influence of pain hypervigilance on the ERP was not significantly affected by chronic pain status (interaction PVAQ*chronic pain status; Figure 4A). Three or more consecutive 20-ms ERFIAs were seen in the latency range 860-920 ms only for P4 and T4. With respect to the separate influence of chronic pain status and PVAQ, chronic pain status affected the habituation course independently of pain hypervigilance at several time latencies (Figure 4B and C). Moreover, pain hypervigilance significantly impacted the habituation course independently of chronic pain status (Figure 4D and E).

\section{Discussion}

In this study, the association between pain hypervigilance and habituation to pain, as measured using cortical responses to 25 intracutaneously delivered painful stimuli, was examined in 33 pain-free and 33 chronic pain participants. Our hypothesis that the effect of pain status on habituation would be further impacted by pain hypervigilance was not supported. Thus, the association between pain status and habituation does not appear to be moderated by pain hypervigilance. Further, the influence of pain hypervigilance and chronic pain status on habituation independently impacted the cortical processing of pain, suggesting two separate mechanisms.

\section{Chronic pain status, hypervigilance, and their relationship with habituation}

These findings imply that chronic pain and pain hypervigilance influence habituation in an additive - rather than synergistic - manner, prompting the study of whether other psychological factors, such as anxiety and depression, act similarly on habituation in chronic pain. Greater insight into these psychological interactions might improve targeted interventions for chronic pain.

Chronic pain status affected linear habituation and dishabituation (quadratic function) between 220 and $260 \mathrm{~ms}$ for nearly all electrodes, consistent with other studies in which impaired habituation was observed in the $\mathrm{P} 2$ region in chronic pain. ${ }^{15,16}$ In addition, chronic pain had a robust

Table 2 Pain locations in the chronic pain group

\begin{tabular}{llllllll}
\hline Location & Abdomen & Back & Thorax & $\begin{array}{l}\text { Lower } \\
\text { extremities }\end{array}$ & $\begin{array}{l}\text { Upper } \\
\text { extremities }\end{array}$ & $\begin{array}{l}\text { Neck and } \\
\text { shoulder }\end{array}$ & Head/face \\
\hline $\mathrm{N}(\%)$ & $4(12 \%)$ & $9(27 \%)$ & $2(6 \%)$ & $12(36 \%)$ & $7(21 \%)$ & $13(36 \%)$ & $3(9 \%)$ \\
\hline
\end{tabular}

Note: Note that 13 subjects suffered from pain in more than one location. 

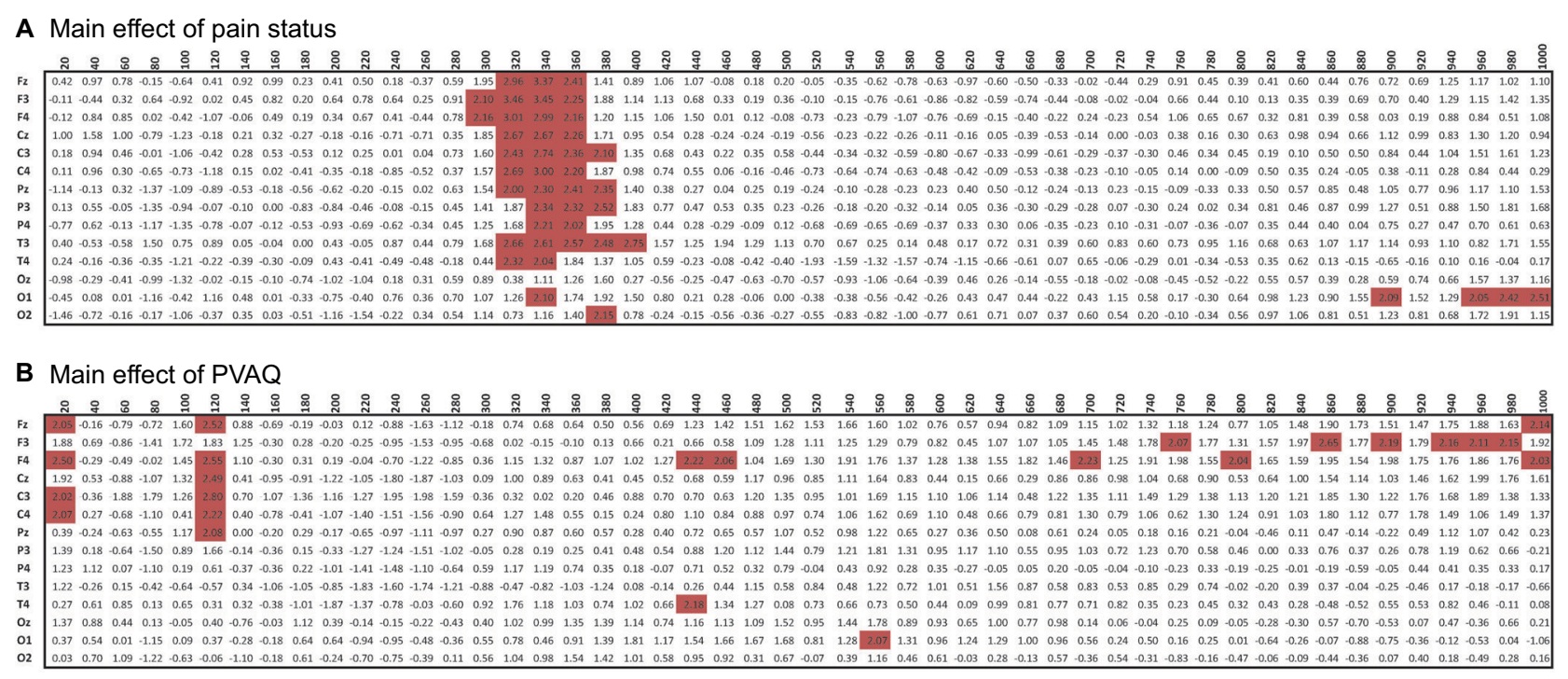

\section{Main effect of linear habituation}

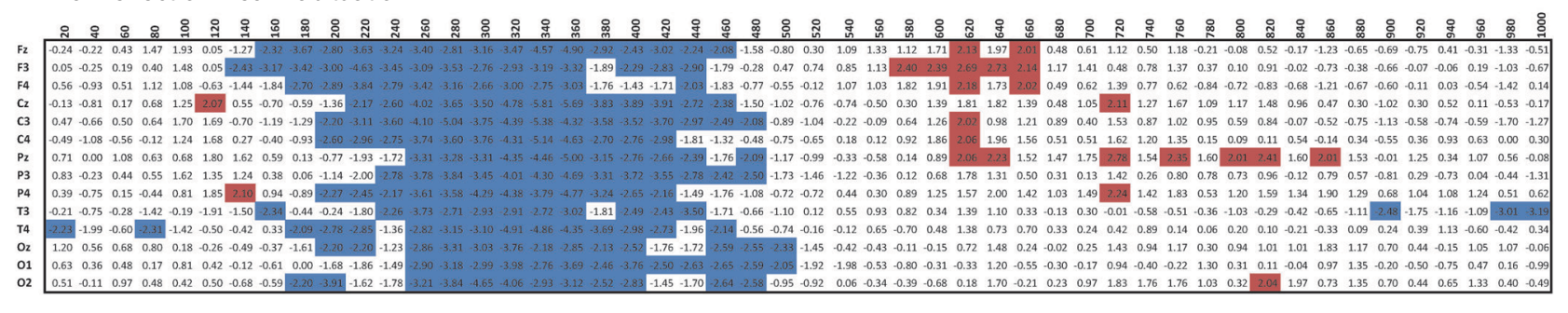

D Main effect of quadratic habituation

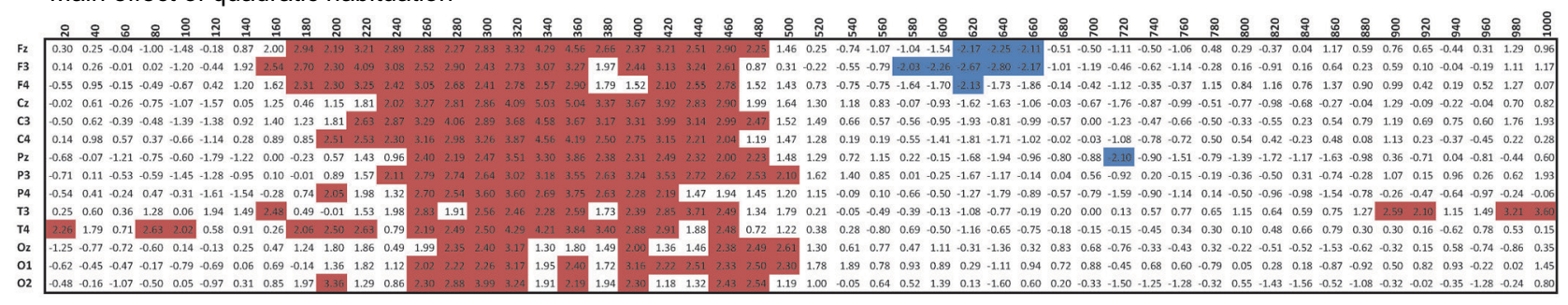

Figure 3 ERFIA predictor blots of the variables pain status (A), PVAQ (B), linear habituation (C) and quadratic habituation (D) of the main multilevel model, and rows display cranial locations. Cells with significant results are colored $(P<0.05)$, and the plus or minus sign expresses the direction of the relationship. Red cells indicate a positive association, and blue cells denote a negative association.

Abbreviations: ERIFIA, event-related fixed interval area; PVAQ, Pain Hypervigilance and Awareness Questionnaire.

effect on habituation between 580 and $640 \mathrm{~ms}$ in the frontal electrodes (F3, F4, and Fz), in contrast to a previous study that examined the influence of chronic low-back pain in 150 electrical stimuli with five intensities (Figure 4). ${ }^{13}$ In our study, the influence of group on habituation was noted primarily from 340 to $460 \mathrm{~ms}$.

There are several explanations for this disparity between studies. For example, the pain-rating protocols differed, and habituation courses over 150 stimuli might be dissimilar when compared with 25 stimuli. Further, five stimulus intensities were used in the previous study instead of one, as in our report. In the former, the previous stimulus intensity (prior to the current stimulus) affected the cortical processing of the current stimulus intensity tremendously in the region from 400 to $680 \mathrm{~ms}$, implying that habituation courses are affected by intensity processes. Consequently, the advantage of the use of a single stimulus intensity over 25 trials is that habituation can be assessed without the interference of stimulus processes. Conversely, in daily practice, individuals are generally confronted with mixed intensities. Thus, the current model is not as generalizable to daily life as a model with mixed intensities.

The effect of pain hypervigilance on habituation was observed primarily from 480 to $820 \mathrm{~ms}$ poststimulus for electrodes on the right side (F4, C4, P4, T4, and $\mathrm{O} 2$ ) and centrally $(\mathrm{Cz}$ and $\mathrm{Pz}$ ), whereas the effect of pain status on habituation 
A Interaction PVAQ with pain status

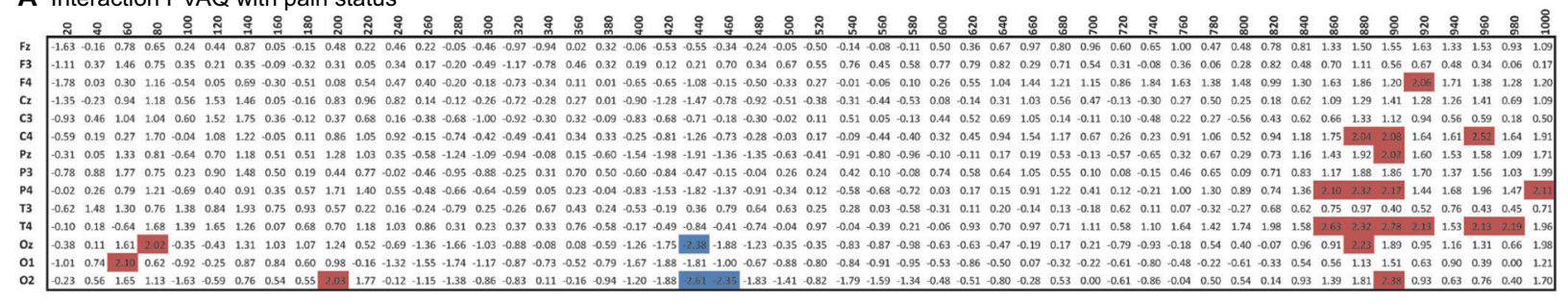

B Interaction of pain status with linear habituation

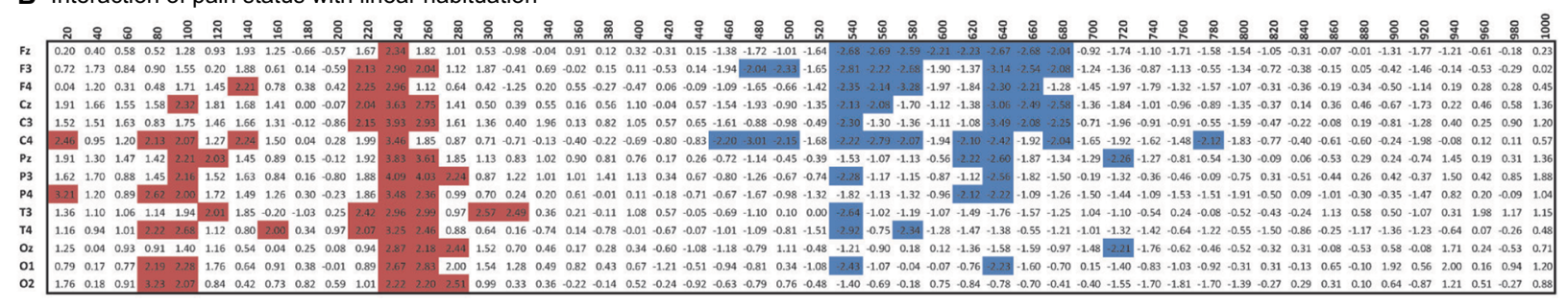

C Interaction of pain status with quadratic habituation

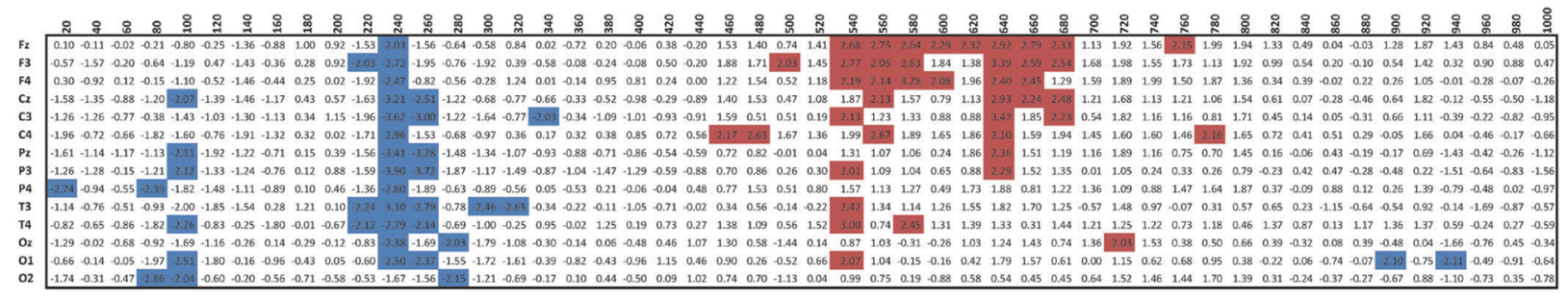

D Interaction of PVAQ with linear habituation

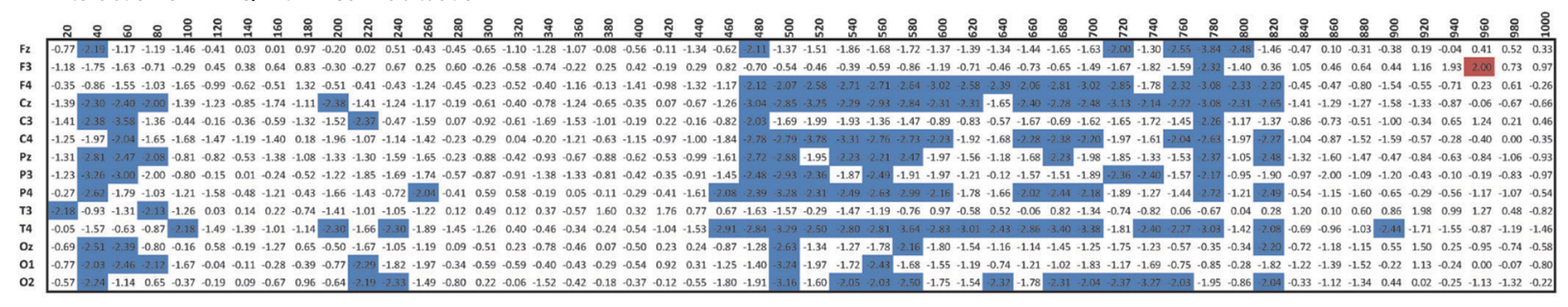

E Interaction of PVAQ with quadratic habituation

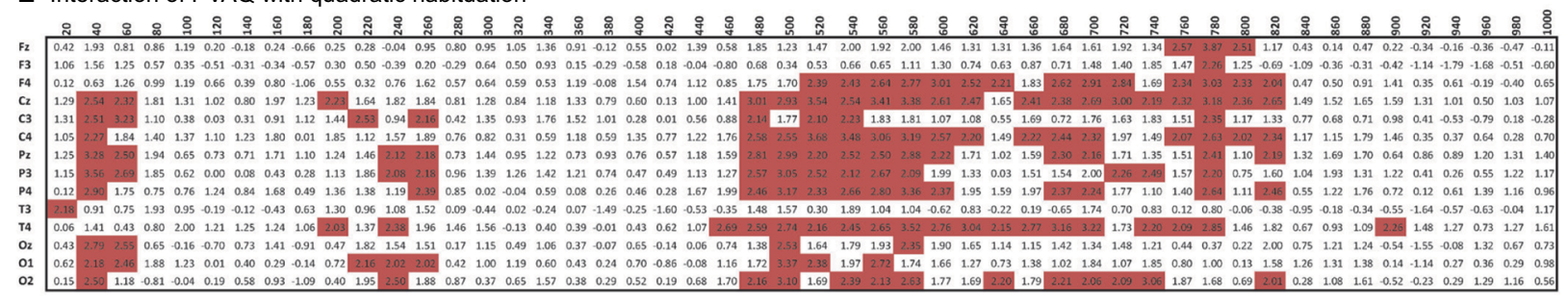

Figure 4 ERFIA predictor blots of the five interactions (A to E). Columns represent consecutive 20-ms ERFIAs, and rows display cranial locations. Cells with significant results are colored $(P<0.05)$, and the plus or minus sign expresses the direction of the relationship. Red cells reflect a positive association, and blue cells denote a negative association.

Abbreviations: ERIFIA, event-related fixed interval area; PVAQ, Pain Hypervigilance and Awareness Questionnaire.

was seen mainly in the frontal areas, indicating that disparate cortical pathways influence habituation (Figure 4). Although EEG has excellent temporal resolution, its spatial resolution is limited. To confirm that these effects have different pathways, more research is needed in which EEG is combined with other modalities with good spatial resolution, such as functional magnetic resonance imaging (fMRI).
In our study, however, the interaction effects of pain hypervigilance on habituation were more pronounced and occurred in a broader latency window, compared with a study of only healthy participants (Vossen 2016, unpublished data). In the current model, the influence of pain hypervigilance on habituation was independent of pain status, requiring other explanations. Because our study consisted of 66 participants 
versus the 46 participants in the other report, the statistical power might be an issue. Moreover, unknown factors, which have not been incorporated into the model, might have played a role. For example, psychological factors, such as anxiety and depression, might influence the association between pain hypervigilance and habituation. High levels of catastrophic thinking about pain are related to a greater fear of pain and attentional bias. ${ }^{32,33}$ A meta-analysis demonstrated that threat-related attention biases exist in several populations with high anxiety levels. ${ }^{34}$ In addition, negative affect can amplify pain-related fear in chronic pain sufferers. ${ }^{17,35}$ Future research in larger populations is required to determine how these psychological factors alter the mechanism of habituation in the cortical processing of pain.

\section{NRS courses over 25 stimuli}

NRS scores decreased significantly over 25 trials, but this decline was not modified by pain hypervigilance or pain status. In the present study, pain hypervigilance and pain status influenced the habituation to cortical responses of painful stimuli but not their subsequent behavioral responses, as expressed by the NRS scores. One argument is that the pain experience is also affected by the threat value of the stimulus. ${ }^{32,36-38}$ It is conceivable that the threat value of stimuli in an experimental setting is low, because the participant is able to stop the protocol at any time. Further, the NRS merely measures the intensity aspect of pain stimuli; affective and social components are not taken into account. Discrepancies between neurophysiological effects and measures of overt behavior, such as the NRS, are common. With regard to the multidimensionality of the perception of pain, future research should address the challenge that remains in translating the effects at the cortical level to those on behavioral responses.

\section{Limitations}

One of the limitations of this study is the heterogeneity of the chronic pain group, which consisted of a sample that was derived from the general population. Although the causes and locations of pain differed, all participants had suffered from pain for at least 6 months. Nevertheless, this study should be replicated for specific pain populations. In the context of external validity, our results might be more generalizable to the general population, as compared with specific pain populations. Furthermore, chronic pain is increasingly considered a distinct nosological category. Thus, central mechanisms, such as habituation, might be comparable between chronic pain populations. ${ }^{39-42}$ Another issue pertains to the limited number of cranial locations (14) in this study; ideally, more locations should be examined.

The habituation protocol comprised 25 stimuli that were $25 \%$ above the pain threshold. Additional habituation sessions with pain-free stimuli and stimuli that exceed the pain threshold by $25 \%$ should be implemented to assess whether the influence of pain status and psychological factors on habituation is associated with stimulus intensity. Further, the existence of a dose-response relationship and the factors that primarily or solely influence habituation to pain should be determined. In general, habituation is more robust with weaker (and, thus, non-painful) stimuli. ${ }^{43,44}$ Thus, the effect of pain status and pain hypervigilance on habituation might be less pronounced at lower pain intensities.

This study evaluated the associations among three variables - pain status, pain hypervigilance, and habituation using a cross-sectional approach. To determine whether and how such factors as pain hypervigilance and the degree of habituation contribute to the development of chronic pain, trials with a longitudinal design are required. The current cross-sectional approach could not address these questions. However, our analyses shed light on the relationship between pain status, pain hypervigilance, and habituation.

\section{New opportunities}

The ERFIA multilevel method has engendered new opportunities to study the influence of psychological factors on the mechanism of habituation to pain. This study provides evidence of the effects of pain hypervigilance - a psychological construct - in non-peak-related areas. Based on our findings, the range of $480-820 \mathrm{~ms}$ is notable with regard to the study of other psychological factors that are linked to habituation in pain. Preferably, such research should be accompanied by fMRI to obtain a greater understanding of temporal and spatial information with regard to pain processing.

For example, Smith et al found that resilience, optimism, and purpose in life facilitated habituation of heat pain and cold pressor pain. ${ }^{11}$ Because habituation can be seen as a protective mechanism and is believed to be mediated by higher cognitive factors, more insight into "positive" and "negative" psychological factors that facilitate or inhibit habituation can guide the development of psychological treatments, tailored to specific needs.

\section{Conclusion}

Pain hypervigilance and chronic pain independently - not synergistically - impact habituation to painful stimuli. The range of 480-820 ms poststimulus may be of importance in 
studying the influence of psychological factors on the cortical processing of pain.

\section{Acknowledgments}

The authors are grateful to Marga Schnitzeler, for recruiting participants and conducting the EEG measurements and data management, and to Dr. Wolfgang Viechtbauer, for providing statistical advice (both from the Department of Psychiatry and Neuropsychology, Maastricht University Medical Centre).

\section{Disclosure}

The authors report no conflicts of interest in this work.

\section{References}

1. Loeser JD, Melzack R. Pain: an overview. Lancet. 1999;353(9164): 1607-1609.

2. Treede RD, Rief W, Barke A, et al. A classification of chronic pain for ICD-11. Pain. 2015;156(6):1003-1007.

3. Woolf CJ. Central sensitization: implications for the diagnosis and treatment of pain. Pain. 2011;152(3 Suppl):S2-S15.

4. Slepian PM, France CR, Rhudy JL, et al. Behavioral inhibition and behavioral activation are related to habituation of nociceptive flexion reflex, but not pain ratings. J Pain. 2017;18(3):349-358.

5. Bingel U, Schoell E, Herken W, Büchel C, May A. Habituation to painful stimulation involves the antinociceptive system. Pain. 2007; 131(1-2):21-30.

6. Bingel U, Tracey I. Imaging CNS modulation of pain in humans. Physiology (Bathesda). 2008;23(6):371-380.

7. Thompson RF, Spencer WA. Habituation: a model phenomenon for the study of neuronal substrates of behavior. Psychol Rev. 1966;73(1):16-43.

8. Rankin CH, Abrams T, Barry RJ, et al. Habituation revisited: an updated and revised description of the behavioral characteristics of habituation. Neurobiol Learn Mem. 2009;92(2):135-138.

9. Kandel ER. The biology of memory: a forty-year perspective. J Neurosci. 2009;29(41):12748-12756.

10. Rennefeld C, Wiech K, Schoell ED, Lorenz J, Bingel U. Habituation to pain: further support for a central component. Pain. 2010;148(3): 503-508.

11. Smith BW, Tooley EM, Montague EQ, Robinson AE, Cosper CJ, Mullins PG. The role of resilience and purpose in life in habituation to heat and cold pain. J Pain. 2009;10(5):493-500.

12. Schuh-Hofer S, Baumgärtner U, Treede RD. Effect of sleep deprivation on the electrophysiological signature of habituation to noxious laser stimuli. Eur J Pain. 2015;19(8):1197-1209.

13. Vossen CJ, Vossen HG, Joosten EA, van Os J, Lousberg R. Does habituation differ in chronic low back pain subjects compared to pain-free controls? A cross-sectional pain rating ERP study reanalyzed with the ERFIA multilevel method. Medicine (Baltimore). 2015;94(19):e865.

14. Valeriani M, de Tommaso M, Restuccia D, et al. Reduced habituation to experimental pain in migraine patients: a $\mathrm{CO}(2)$ laser evoked potential study. Pain. 2003;105(1-2):57-64.

15. de Tommaso M, Federici A, Santostasi R, et al. Laser-evoked potentials habituation in fibromyalgia. J Pain. 2011;12(1):116-124.

16. Smith BW, Tooley EM, Montague EQ, Robinson AE, Cosper CJ, Mullins PG. Habituation and sensitization to heat and cold pain in women with fibromyalgia and healthy controls. Pain. 2008;140(3):420-428.

17. Crombez G, Eccleston C, Baeyens F, van Houdenhove B, van den Broeck A. Attention to chronic pain is dependent upon pain-related fear. J Psychosom Res. 1999;47(5):403-410.
18. McCracken LM. "Attention" to pain in persons with chronic pain: a behavioral approach. Behav Ther. 1997;28(2):271-284.

19. Goubert L, Crombez G, Van Damme S. The role of neuroticism, pain catastrophizing and pain-related fear in vigilance to pain: a structural equations approach. Pain. 2004;107(3):234-241.

20. Lautenbacher S, Huber C, Kunz M, et al. Hypervigilance as predictor of postoperative acute pain: its predictive potency compared with experimental pain sensitivity, cortisol reactivity, and affective state. Clin J Pain. 2009;25(2):92-100.

21. Baum C, Huber C, Schneider R, Lautenbacher S. Prediction of experimental pain sensitivity by attention to pain-related stimuli in healthy individuals. Percept Mot Skills. 2011;112(3):926-946.

22. Rollman GB. Perspectives on hypervigilance. Pain. 2009;141(3):183-184.

23. Vossen CJ, Vossen HG, Marcus MA, Van Os J, Lousberg R. Introducing the event related fixed interval area (ERFIA) multilevel technique: a method to analyze the complete epoch of event-related potentials at single trial level. PLoS One. 2013;8(11):e79905.

24. Vossen H, Van Breukelen G, Hermens H, Van Os J, Lousberg R. More potential in statistical analyses of event-related potentials: a mixed regression approach. Int J Methods Psychiatr Res. 2011;20(3):e56-e68.

25. Aaronson NK, Muller M, Cohen PD, et al. Translation, validation, and norming of the Dutch language version of the SF-36 health survey in community and chronic disease populations. J Clin Epidemiol. 1998;51(11):1055-1068.

26. Daut RL, Cleeland CS, Flanery RC. Development of the Wisconsin Brief Pain Questionnaire to assess pain in cancer and other diseases. Pain. 1983;17(2):197-210.

27. Shahid A, Wilkinson K, Marcu S, Shapiro CM. Brief Pain Inventory (BPI). In: STOP, THAT and One Hundred Other Sleep Scales. Vol 1100. New York, NY: Springer New York; 2011:81-88.

28. Roelofs J, Peters ML, Muris P, Vlaeyen JW. Dutch version of the Pain Vigilance and Awareness Questionnaire: validity and reliability in a pain-free population. Behav Res Ther. 2002;40(9):1081-1090.

29. Roelofs J, Peters ML, McCracken L, Vlaeyen JW. The pain vigilance and awareness questionnaire (PVAQ): further psychometric evaluation in fibromyalgia and other chronic pain syndromes. Pain. 2003;101(3): 299-306.

30. Bromm B, Lorenz J. Neurophysiological evaluation of pain. Electroencephalogr Clin Neurophysiol. 1998;107(4):227-253.

31. Klem GH, Lüders HO, Jasper HH, Elger C. The ten-twenty electrode system of the International Federation. The International Federation of Clinical Neurophysiology. Electroencephalogr Clin Neurophysiol Suppl. 1999;52:3-6.

32. Crombez G, Eccleston C, Baeyens F, Eelen P. When somatic information threatens, catastrophic thinking enhances attentional interference. Pain. 1998;75(2-3):187-198.

33. Crombez G, Eccleston C, Van den Broeck A, Goubert L, Van Houdenhove B. Hypervigilance to pain in fibromyalgia: the mediating role of pain intensity and catastrophic thinking about pain. Clin J Pain. 2004;20(2):98-102.

34. Bar-Haim Y, Lamy D, Pergamin L, Bakermans-Kranenburg MJ, van IJzendoorn MH. Threat-related attentional bias in anxious and nonanxious individuals: a meta-analytic study. Psychol Bull. 2007; 133(1):1-24.

35. Wong WS, Lam HM, Chen PP, et al. The fear-avoidance model of chronic pain: assessing the role of neuroticism and negative affect in pain catastrophizing using structural equation modeling. Int J Behav Med. 2015;22(1):118-131.

36. Aldrich S, Eccleston C, Crombez G. Worrying about chronic pain: vigilance to threat and misdirected problem solving. Behav Res Ther. 2000;38(5):457-470.

37. Van Damme S, Crombez G, Eccleston C, Koster EH. Hypervigilance to learned pain signals: a componential analysis. J Pain. 2006;7(5):346-357.

38. Moore DJ, Keogh E, Eccleston C. The effect of threat on attentional interruption by pain. Pain. 2013;154(1):82-88. 
39. Flor H, Braun C, Elbert T, Birbaumer N. Extensive reorganization of primary somatosensory cortex in chronic back pain patients. $\mathrm{Neurosci}$ Lett. 1997;224(1):5-8.

40. Tracey I, Bushnell MC. How neuroimaging studies have challenged us to rethink: is chronic pain a disease? J Pain. 2009;10(11):1113-1120.

41. Phillips K, Clauw DJ. Central pain mechanisms in chronic pain statesmaybe it is all in their head. Best Pract Res Clin Rheumatol. 2011;25(2): $141-154$.
42. Apkarian AV, Hashmi JA, Baliki MN. Pain and the brain: specificity and plasticity of the brain in clinical chronic pain. Pain. 2011;152(3 Suppl): S49-S64.

43. von Dincklage F, Olbrich H, Baars JH, Rehberg B. Habituation of the nociceptive flexion reflex is dependent on inter-stimulus interval and stimulus intensity. J Clin Neurosci. 2013;20(6):848-850.

44. Thompson RF. Habituation: a history. Neurobiol Learn Mem. 2009;92(2):127-134.
Journal of Pain Research

\section{Publish your work in this journal}

The Journal of Pain Research is an international, peer reviewed, open access, online journal that welcomes laboratory and clinical findings in the fields of pain research and the prevention and management of pain. Original research, reviews, symposium reports, hypothesis formation and commentaries are all considered for publication.
The manuscript management system is completely online and includes a very quick and fair peer-review system, which is all easy to use. Visit http://www.dovepress.com/testimonials.php to read real quotes from published authors. 